\title{
Field theoretical study of a spin-1/2 ladder with unequal chain exchanges
}

\author{
Sujit Sarkar ${ }^{1}$ and Diptiman Sen ${ }^{2}$ \\ ${ }^{1}$ Max-Planck-Institute for the Physics of Complex Systems, Noethnitzer Strasse 38, 01187 Dresden, Germany \\ ${ }^{2}$ Centre for Theoretical Studies, Indian Institute of Science, Bangalore 560012, India
}

(Received 19 November 2001; published 18 April 2002)

\begin{abstract}
We study the low-energy properties of a Heisenberg spin-1/2 zigzag ladder with different exchange constants on the two chains. Using a nonlinear $\sigma$-model field theory and Abelian bosonization, we find that the excitations are gapless, with a finite spin-wave velocity, if the values of the chain exchanges are small. If the chain exchanges are large, the system is gapped, and the energy spectra of the kink and antikink excitations are different from each other.
\end{abstract}

DOI: 10.1103/PhysRevB.65.172408

PACS number(s): 75.10.Jm

\section{INTRODUCTION}

For the last several decades, one-dimensional and quasione-dimensional quantum spin systems have been studied extensively. Experimentally, many such systems are known to present a wide range of unusual properties, and a variety of analytical and numerical techniques exist for studying these systems theoretically. The three observations which make the low-dimensional spin systems particularly intersting are (i) Haldane's conjecture for one-dimensional antiferromagnetic spin systems, ${ }^{1}$ (ii) the discovery of hightemperature superconductivity and its magnetic properties at low doping, ${ }^{2}$ and (iii) the discovery of ladder materials. ${ }^{3}$

In spin ladders, two or more spin chains interact with each other. For ladders with a railroad geometry, it has been observed that spin-1/2 systems with an even number of legs are gapped, while systems with an odd number of chains have gapless excitations. ${ }^{4,5}$ However, the frustrated zigzag ladder shows a gapless spin liquid state or a gapped dimer state, depending on the ratio of the exchanges of the rungs to the chains. ${ }^{7}$

Another interesting kind of system is the spin-Peierls system such as $\mathrm{CuGeO}_{3}$. The ground state and the lowtemperature thermodynamic properties of this have been studied in great detail, and it has been shown that there is a spontaneous dimerization of the nearest-neighbor interaction below a particular temperature. ${ }^{6}$

Relatively less is known about a spin ladder with asymmetry in the chains. An extreme case of this situation, i.e., when a chain is absent from the Fig. 1 (sawtooth chain), has been studied by two groups. ${ }^{8}$ The ground state is like that of the Majumdar-Ghosh model, ${ }^{9}$ except that the two kinds of low-energy excitations (kinks and antikinks) which interpolate between the two degenerate ground states have different excitation spectra.

In this paper, we study a two-chain ladder system with unequal exchange constants of the chains, rather than a dimerization in the rungs. Recently, Chen et al ${ }^{10}$ studied this problem. We will discuss their results below. The plan of the paper is as follows. In Sec. II, we will analyze the problem using the nonlinear $\sigma$-model (NLSM) field theory. Section III will discuss the Abelian bosonization approach.

\section{NONLINEAR $\sigma$-MODEL STUDY}

In this section we study the NLSM theory of our model, which is shown schematically in Fig. 1. The system can be viewed either as a ladder with unequal exchanges on the two chains, or as a single chain with unequal next-nearestneighbor exchanges. The Hamiltonian is

$$
H=\sum_{n}\left[J_{1} \vec{S}_{n} \cdot \vec{S}_{n+1}+J_{2}\left(1-(-1)^{n} \delta\right) \vec{S}_{n} \cdot \vec{S}_{n+2}\right],
$$

where $n$ is the site index, $J_{1}, J_{2} \geqslant 0$, and $0 \leqslant \delta \leqslant 1$. If we view the system as two chains, then the lower (upper) chain contains the odd (even) numbered sites.

To begin the analysis, we first find the configuration of the spins in the classical ground state of the model. For small values of $J_{2} / J_{1}$, neighboring spins are aligned antiparallel to each other; this is called the Neel phase. We can then derive a NLSM field theory which describes the low-energy and long-wavelength excitations. In the Neel phase, this is given by an $O(3)$ NLSM with a topological term. We define two fields $\vec{\phi}_{n}$ and $\vec{l}_{n}$ as a linear combination of two spins as

$$
\begin{aligned}
& \vec{\phi}_{n}=\frac{\vec{S}_{2 n-1}-\vec{S}_{2 n}}{2 S}, \\
& \vec{l}_{n}=\frac{\vec{S}_{2 n-1}+\vec{S}_{2 n}}{2 a},
\end{aligned}
$$

where $a$ is the lattice spacing. One can check that $\vec{\phi}_{n} \cdot \vec{l}_{n}$ $=0$, and

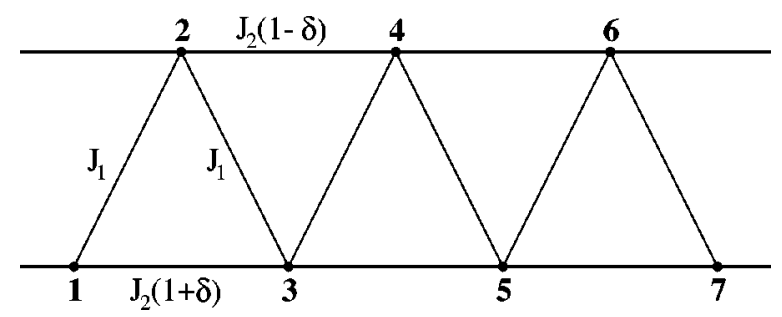

FIG. 1. Schematic diagram of a spin ladder with unequal chain exchanges. 


$$
\vec{\phi}_{n}^{2}=1+\frac{1}{S}-\frac{a^{2} \vec{l}_{n}^{2}}{S^{2}} .
$$

Thus $\vec{\phi}_{n}$ becomes a unit vector in the large- $S$ limit; note that this fact depends crucially on the antiparallel arrangement of neighboring spins in the classical ground state in the Neel phase. The unit cell of this ground state is labeled by an integer $n$, and it contains the sites $2 n-1$ and $2 n$, respectively; the length of a unit cell is $2 a$.

The fields $\vec{\phi}_{n}$ and $\vec{l}_{n}$ satisfy the commutation relations

$$
\left[\vec{l}_{m a}, \vec{\phi}_{n b}\right]=\frac{i}{2 a} \delta_{m n} \sum_{c} \epsilon_{a b c} \vec{\phi}_{n c},
$$

where $m$ and $n$ are the unit cell labels; $a, b$, and $c$ denote the $x, y$, and $z$ components of the field; and $\epsilon_{x y z}$ is the completely antisymmetric tensor with $\epsilon_{x y z}=1$. This relation enables us to write $\vec{l}_{n}=\vec{\phi}_{n} \times \vec{\Pi}_{n}$, where the vector $\vec{\Pi}$ is canonically conjugate to $\vec{\phi}$, namely,

$$
\left[\phi_{m a}, \Pi_{n b}\right]=\frac{i}{2 a} \delta_{m n} \delta_{a b} .
$$

To define the continuum limit of this theory, we introduce a spatial coordinate $x$ which is equal to $2 n a$ at the location of the $n$th unit cell. Summations are then replaced by integrals, i.e., $\Sigma_{n} \rightarrow \int d x /(2 a)$.

Since $\vec{\phi}_{n}$ is a unit vector, $\dot{\vec{\phi}}$ and $\vec{\phi}^{\prime}$ are orthogonal to $\vec{\phi}$. In the low-energy and long-wavelength limit, the dominant terms in the Hamiltonian are those which have second-order space-time derivatives of $\vec{\phi}$ and first-order derivatives of $\vec{l}$ (since $\vec{l}$ contains first-order derivatives of $\vec{\phi}$ ). ${ }^{11,12}$ We expand the fields $\vec{\phi}_{n+1}=\vec{\phi}(x+2 a)$ and $\vec{l}_{n+1}=\vec{l}(x+2 a)$, where $x$ $=2 n a$, as

$$
\begin{gathered}
\vec{\phi}(x+2 a)=\vec{\phi}(x)+2 a \vec{\phi}^{\prime}(x)+2 a^{2} \vec{\phi}^{\prime \prime}(x)+\cdots, \\
\vec{l}(x+2 a)=\vec{l}(x)+2 a \vec{l}^{\prime}(x)+\cdots
\end{gathered}
$$

Using Eqs. (2), (3), and (6), we obtain the Hamiltonian

$$
H=\int d x\left[\frac{c g^{2}}{2}\left(\vec{l}+\frac{\theta}{4 \pi} \vec{\phi}^{\prime}\right)^{2}+\frac{c}{2 g^{2}} \vec{\phi}^{\prime 2}\right],
$$

where

$$
\begin{gathered}
c=2 J_{1} S a \sqrt{1-4 J_{2} / J_{1}}, \\
g^{2}=\frac{2}{S \sqrt{1-4 J_{2} / J_{1}}}, \\
\theta=2 \pi S .
\end{gathered}
$$

Note that the values of $c, g^{2}$ and $\theta$ turn out to be independent of $\delta$ in this approach. Further, this NLSM is valid only if $J_{2} / J_{1}<1 / 4$. For $J_{2} / J_{1}>1 / 4$, a different NLSM is required because the configuration of the spins in the classical ground state is then a spiral in a plane rather than antiparallel arrangement of neighboring spins. The order parameter of the NLSM in the spiral phase is a $\mathrm{SO}(3)$ matrix instead of a unit vector. $^{11}$

One can find the energy-momentum dispersion relation of the form $\omega=c|k|$, where $\mathrm{c}$ is the spin-wave velocity, by considering small fluctuations around $\vec{\phi}=(0,0,1)$, and expanding the Hamiltonian in Eq. (7) to second order in those fluctuations. Similarly one can find the strength of the interaction between the spin waves, $g^{2}$, by expanding the Hamiltonian to fourth order in the fluctuations.

From Eq. (8), we see that the coefficient of the topological term $\theta=\pi$ for $S=1 / 2$. This implies that there is no gap in the low-energy excitation spectrum. This result is different from that of the zigzag ladder with a dimerization in the nearest-neighbor interaction, i.e., with a term like $J_{1}(-1)^{n} \vec{S}_{n} \cdot \vec{S}_{n+1}$. In this case $\theta=2 \pi S(1-\delta)$ is different from $\pi$ for $S=1 / 2,{ }^{12,13}$ and the the low-energy excitations are gapped.

\section{ABELIAN BOSONIZATION STUDY}

We now study the low-energy spectrum using Abelian bosonization. We write the Hamiltonian as

$$
H=H_{1}+H_{2}+H_{2 \delta},
$$

where

$$
\begin{gathered}
H_{1}=J_{1} \sum_{n} \vec{S}_{n} \cdot \vec{S}_{n+1}, \\
H_{2}=J_{2} \sum_{n} \vec{S}_{n} \cdot \vec{S}_{n+2}, \\
H_{2 \delta}=-J_{2} \delta \sum_{n}(-1)^{n} \vec{S}_{n} \cdot \vec{S}_{n+2} .
\end{gathered}
$$

We then convert this to a Hamiltonian of spinless fermions using the Jordan-Wigner transformation. The relations between the spin and the electron creation and annihilation operators are

$$
\begin{gathered}
S_{n}^{z}=\psi_{n}^{\dagger} \psi_{n}-1 / 2, \\
S_{n}^{-}=(-1)^{n} \psi_{n} \exp \left[i \pi \sum_{j=-\infty}^{n-1} n_{j}\right], \\
S_{n}^{+}=(-1)^{n} \psi_{n}^{\dagger} \exp \left[-i \pi \sum_{j=-\infty}^{n-1} n_{j}\right],
\end{gathered}
$$

where $n_{j}=\psi_{j}^{\dagger} \psi_{j}$ is the fermion number at site $j$. The Hamiltonians in Eqs. (10) then become

$$
\begin{aligned}
H_{1}= & -\frac{J_{1}}{2} \sum_{n}\left(\psi_{n+1}^{\dagger} \psi_{n}+\psi_{n}^{\dagger} \psi_{n+1}\right) \\
& +J_{1} \sum_{n}\left(\psi_{n}^{\dagger} \psi_{n}-1 / 2\right)\left(\psi_{n+1}^{\dagger} \psi_{n+1}-1 / 2\right),
\end{aligned}
$$




$$
\begin{gathered}
H_{2}=J_{2} \sum_{n}\left(\psi_{n+2}^{\dagger} \psi_{n}+\text { h.c. }\right)\left(\psi_{n+1}^{\dagger} \psi_{n+1}-1 / 2\right) \\
\quad+J_{2} \sum_{n}\left(\psi_{n}^{\dagger} \psi_{n}-1 / 2\right)\left(\psi_{n+2}^{\dagger} \psi_{n+2}-1 / 2\right), \\
H_{2 \delta}=-J_{2} \delta \sum_{n}(-1)^{n}\left(\psi_{n+2}^{\dagger} \psi_{n}+\text { h.c. }\right)\left(\psi_{n+1}^{\dagger} \psi_{n+1}-1 / 2\right) \\
-J_{2} \delta \sum_{n}(-1)^{n}\left(\psi_{n}^{\dagger} \psi_{n}-1 / 2\right)\left(\psi_{n+2}^{\dagger} \psi_{n+2}-1 / 2\right) .
\end{gathered}
$$

We will assume below that $J_{1} \gg J_{2}$. In the absence of a magnetic field, the two Fermi points lie at $k_{F}= \pm \pi / 2$. We linearize the energy spectrum around these points, and express the lattice operators in terms of two continuum fields $R$ and $L$ which vary slowly on the scale of a lattice spacing,

$$
\psi_{n}=\sqrt{a}\left[i^{n} R(n)+(-i)^{n} L(n)\right],
$$

where $R$ and $L$ describe the second-quantized fields of rightand left-moving fermions, respectively. Now we bosonize our Hamiltonian following the standard procedure. The basic relations used to obtain the bosonized Hamiltonian are ${ }^{14}$

$$
S^{z}(x)=a\left[\rho(x)+(-1)^{j} M(x)\right],
$$

where the fermion density $\rho(x)=: R^{\dagger}(x) R(x)$ : $+: L^{\dagger}(x) L(x)$ : and the mass operator $M(x)=: R^{\dagger}(x) L(x)$ : $+: L^{\dagger}(x) R(x)$ :. (The double dots denote normal ordering). The bosonized expressions for $\rho$ and $M$ are given by

$$
\begin{gathered}
\rho(x)=-\frac{1}{\sqrt{\pi}} \partial_{x} \phi(x), \\
M(x)=\frac{1}{\pi a} \cos [2 \sqrt{\pi} \phi(x)] .
\end{gathered}
$$

The bosonized version of $H_{1}$ is known to be

$$
H_{1}=\int d x\left[\frac{v_{1} K}{2} \Pi^{2}+\frac{v_{1}}{2 K}\left(\partial_{x} \phi\right)^{2}+\frac{v_{1}}{(\pi a)^{2}}: \cos (4 \sqrt{\pi} \phi):\right],
$$

where $v_{1}=\pi J_{1} a / 2$ is the spin-wave velocity, and $K=1 / 2$ is the bosonization interaction parameter for the isotropic spin$1 / 2$ antiferromagnet. The last term in Eq. (18) is marginal, and is known to have no effect at long distances in the sense of the renormalization group (RG). ${ }^{14}$ Using Eqs. (13), (15), and (16), we obtain the following expression for $H_{2}$, where we have ignored terms of the order of $a^{4}$ and higher:

$$
\begin{aligned}
H_{2}= & J_{2} a^{2} \sum_{n}\left\{-\left[\rho_{n+1}-(-1)^{n} M_{n+1}\right]\right. \\
& \times\left[\rho_{n}+\rho_{n+2}+(-1)^{n} M_{n}+(-1)^{n} M_{n+2}\right] \\
& \left.+\left(\rho_{n}+(-1)^{n} M_{n}\right)\left(\rho_{n+2}+(-1)^{n} M_{n+2}\right)\right\} .
\end{aligned}
$$

To derive this, we have used Taylor expansions such as

$$
R(n+2)=R(n)+2 a R^{\prime}(n)+2 a^{2} R^{\prime \prime}(n)+\cdots
$$

to write

$$
\begin{aligned}
& R^{\dagger}(n+2) R(n)+R^{\dagger}(n) R(n+2) \\
& \quad=R^{\dagger}(n+2) R(n+2)+R^{\dagger}(n) R(n)+O\left(a^{2}\right) .
\end{aligned}
$$

On keeping only the terms which do not oscillate as $(-1)^{n}$ (which would give zero in the continuum limit) and then expressing the operators $\rho$ and $M$ in the bosonic language, the above expression becomes

$$
H_{2}=v_{2} \int d x\left[-\frac{1}{\pi}\left(\partial_{x} \phi\right)^{2}+\frac{3}{2(\pi a)^{2}} \cos (4 \sqrt{\pi} \phi)\right],
$$

where $v_{2}=J_{2} a$. Both terms in Eq. (22) have a scaling dimension 2, and they are marginal. It is known that they have no effect in the RG sense as long as $J_{2} / J_{1}<0.2411$. $^{7}$ Finally, we consider the bosonized expression for $H_{2 \delta}$. The nonoscillatory terms in this are given by

$$
\begin{aligned}
H_{2 \delta}= & -J_{2} \delta a^{2} \sum_{n}\left[\rho_{n} M_{n+2}+M_{n} \rho_{n+2}-\rho_{n+1}\left(M_{n}+M_{n+2}\right)\right. \\
& \left.+M_{n+1}\left(\rho_{n}+\rho_{n+2}\right)\right] .
\end{aligned}
$$

Now we perform an operator product expansion of the above Hamiltonian. In the limit $z \rightarrow w$, we can use the expansion ${ }^{15}$

$$
\partial_{z} \phi(z): e^{i \beta \phi(w)}:=-\frac{i \beta}{2 K(z-w)}: e^{i \beta \phi(w)}:+: \partial_{z} \phi(z) e^{i \beta \phi(z)} .
$$

( $\beta=2 \sqrt{\pi}$ and $K=1 / 2$ for us.) The first term in Eq. (24) has a scaling dimension of $\beta^{2} K /(4 \pi)=1 / 2$, and is therefore relevant. However, due to the denominator $z-w$ which takes the values $\pm a$ or $\pm 2 a$, we see that the various relevant terms in Eq. (23) cancel each other; for instance, there are cancellations in $\rho_{n} M_{n+2}+M_{n} \rho_{n+2}, \quad \rho_{n+1} M_{n}+M_{n+1} \rho_{n}$, and $\rho_{n+1} M_{n+2}+M_{n+1} \rho_{n+2}$. If this cancellation had not occurred, the bosonized version of $\mathrm{H}_{2 \delta}$ would have contained the relevant operator $\cos (2 \sqrt{\pi} \phi)$ with a scaling dimension of $1 / 2$. However, due to the cancellation, $H_{2 \delta}$ contains no relevant operators with scaling dimensions less than 2 . The second term in Eq. (24) is a total derivative, and its contribution therefore vanishes in the Hamiltonian where it appears inside an integral over all $x$. Thus the system continues to remain gapless (and lies in the same spin-liquid phase as the model described by the nearest-neighbor Hamiltonian $H_{1}$ ) even if $\delta \neq 0$, provided that $J_{2} \ll J_{1}$. This is in contrast to a dimerization in $J_{1}$; in this case Abelian bosonization correctly produces a relevant term which leads to a gapped phase.

Recently Chen et al. ${ }^{10}$ studied the same model as ours using Abelian bosonization followed by a perturbative renormalization-group analysis. They claim that the perturbation due to the $J_{2} \delta$ terms is relevant, but that it does not lead to a gapped phase; instead, they argue that it leads to a different and unusual fixed point where the system is gapless 
and has a vanishing spin velocity. Our study shows that due to some cancellations, the $J_{2} \delta$ terms do not lead to any relevant terms in the continuum theory; namely, the perturbation which might have led to the unusual fixed point of Chen et al. has a coefficient of zero in the present model. Therefore, the system remains gapless with a finite spin velocity if $J_{2}$ is small.

Finally, let us discuss the low-energy excitations of this model when $J_{2}$ becomes larger. In particular, we find that these are given by kinks and antikinks when $J_{2}=J_{1} / 2$, for all values of $\delta$ lying in the range [0,1]. (The Majumdar-Ghosh model is a special case of this where $\delta=0$ ). Then the Hamiltonian in Eq. (1) can be written, up to a constant, as

$$
\begin{aligned}
H= & \frac{J_{1}}{4} \sum_{n}\left[(1+\delta)\left(\vec{S}_{2 n-1}+\vec{S}_{2 n}+\vec{S}_{2 n+1}\right)^{2}\right. \\
& \left.+(1-\delta)\left(\vec{S}_{2 n}+\vec{S}_{2 n+1}+\vec{S}_{2 n+2}\right)^{2}\right] .
\end{aligned}
$$

Hence the ground state is given by a configuration in which the total spin of each triangle is $1 / 2$. Since this can be done either by forming a singlet with the pair of spins $(2 n$ $-1,2 n$ ) for all values of $n$, or by forming a singlet with the pair of spins $(2 n, 2 n+1)$ for all values of $n$, we see that the ground state is doubly degenerate. Let us denote these two ground states by $A$ and $B$ respectively. The lowest-energy excitations (kink and antikink) are formed by interpolating between these two states. The kink has the ground state $A$ on the left and the ground state $B$ on the right, while the antikink has the ground state $B$ on the left and the ground state $A$ on the right. For general values of $\delta$, we find that the kink and antikink dispersions are nondegenerate in contrast with the Majumdar-Ghosh model. ${ }^{8}$ A simple variational calculations gives the kink and antikink dispersions to be $J_{1}(1-\delta)(5$ $+4 \cos k) / 8$ and $J_{1}(1+\delta)(5+4 \cos k) / 8$, respectively. Hence the minimum gap for the kink and antikink excitations are $J_{1}(1-\delta) / 8$ and $J_{1}(1+\delta) / 8$ respectively, occurring at $k=\pi$. This result was also obtained by Chen et al. ${ }^{10}$

To summarize, we have studied the low-lying excitations of a zigzag ladder with unequal chain exchanges. Both the NLSM and the Abelian bosonization show that the system remains gapless if $J_{2} \ll J_{1}$. We have also shown that the system is gapped (with two degenerate ground states) for $J_{2}$ $=J_{1} / 2$. It would be interesting to use numerical techniques like the density-matrix renormalization group ${ }^{16}$ to study the phase diagram of the ground state as a function of the two parameters $J_{2} / J_{1}$ and $\delta$.

\section{ACKNOWLEDGMENTS}

We would like to thank Marco Ameduri for useful discussions. D.S. would like to thank Bruce Normand for helpful comments, and the Max Planck Institute for the Physics of Complex Systems, Dresden for its hospitality during the course of this work.
${ }^{1}$ F. D. M. Haldane, Phys. Rev. Lett. 50, 1153 (1983); Phys. Lett. A 93, 464 (1983); I. Affleck, in Fields, Strings and Critical Phenomena, edited by E. Brezin and J. Zinn-Justin (North-Holland, Amsterdam, 1989).

${ }^{2}$ J. G. Bednorz and K. A. Muller, Z. Phys. B: Condens. Matter 64, 188 (1986).

${ }^{3}$ D. C. Johnston, J. W. Johnson, D. P. Goshorn, and A. J. Jacobson, Phys. Rev. B 35, 219 (1987): Z. Hiroi, M. Azuma, M. Takano, and Y. Bando, J. Solid State Chem. 95, 230 (1991).

${ }^{4}$ E. Dagotto, J. Riera, and D. Scalapino, Phys. Rev. B 45, 5744 (1992); E. Dagotto and T. M. Rice, Science 271, 618 (1996).

${ }^{5}$ G. Chaboussant, M.-H. Julien, Y. Fagot-Revurat, L. P. Levy, C. Berthier, M. Horvatic, and O. Piovesana, Phys. Rev. Lett. 79, 925 (1997)

${ }^{6}$ G. S. Uhrig, Physica B 280, 308 (2000); J. P. Boucher and L. P. Regnault, J. Phys. I 6, 1939 (1996); J. Riera and A. Dobry, Phys. Rev. B 51, 16098 (1995); G. Castilla, S. Chakravarty, and V. J. Emery, Phys. Rev. Lett. 75, 1823 (1995); M. C. Cross and D. S. Fisher, Phys. Rev. B 19, 402 (1979).

${ }^{7}$ S. Eggert, Phys. Rev. B 54, R9612 (1996); K. Okamoto and K. Nomura, Phys. Lett. A 169, 433 (1992); R. Jullien and F. D. M. Haldane, Bull. Am. Phys. Soc. 28, 344 (1983): S. R. White and
I. Affleck, Phys. Rev. B 54, 9862 (1996).

${ }^{8}$ T. Nakamura and K. Kubo, Phys. Rev. B 53, 6393 (1996); D. Sen, B. S. Shastry, R. E. Walstedt, and R. Cava, ibid. 53, 6401 (1996).

${ }^{9}$ C. K. Majumdar and D. K. Ghosh, J. Math. Phys. 10, 1388 (1969); 10, 1399 (1969).

${ }^{10}$ S. Chen, H. Büttner, and J. Voit, Phys. Rev. Lett. 87, 087205 (2001); cond-mat/0201004 (unpublished).

${ }^{11}$ S. Rao and D. Sen, Nucl. Phys. B 424, 547 (1994).

${ }^{12}$ D. Sen, cond-mat/0107082 (unpublished).

${ }^{13}$ G. Sierra, in Strongly Correlated Magnetic and Superconducting Systems, edited by G. Sierra and M. A. Martin-Delgado, Lecture Notes in Physics Vol. 478 (Springer, Berlin, 1997).

${ }^{14}$ A. O. Gogolin, A. A. Nersesyan, and A. M. Tsvelik, Bosonization and Strongly Correlated Systems (Cambridge University Press, Cambridge, 1998).

${ }^{15}$ N. Nagaosa, Quantum Field Theory in Strongly Correlated Electronic Systems (Springer-Verlag, Berlin, 1999); C. J. Efthimiou and D. A. Spector, hep-th/0003190 (unpublished).

${ }^{16}$ S. K. Pati, S. Ramasesha, and D. Sen, cond-mat/0106621 (unpublished). 